\title{
Evaluation of Probe Impedance Due to Thin-Skin Eddy-Current Interaction with Surface Cracks
}

\author{
J. R. Bowler and N. Harfield
}

\begin{abstract}
Crack detection using eddy-current nondestructive testing is often carried out at frequencies such that the skin depth of the induced current is much smaller than the crack dimensions. The induced current then flows in a thin skin at the conductor surface and at the faces of a surface crack. In the case of a crack that acts as an impenetrable barrier to electric current, the electromagnetic field at the crack surface can be represented, at an arbitrary frequency, in terms of a potential which satisfies a two-dimensional Laplace equation. The boundary conditions required in the solution of the Laplace equation have not yet been determined for the general case, but we have derived approximate boundary conditions which are applicable in the thin-skin regime. The conditions derived are valid for cracks in materials of arbitrary permeability. From the harmonic solutions of the Laplace equation, the impedance change of the excitation coil due to the defect has been calculated for cracks in aluminum and ferromagnetic steel. Comparisons between predictions and experimental measurements on rectangular slots show good agreement, thus corroborating the theory and the numerical calculations.
\end{abstract}

Index Terms - Crack, eddy current, Laplace equation.

\section{INTRODUCTION}

$\mathbf{E}$ DDY-CURRENT nondestructive testing is commonly carried out on metals at a single frequency using an induction probe which senses the presence of cracks through changes in its driving point impedance. These tests are often performed at frequencies such that the electromagnetic skin depth is much smaller than the crack depth. Thin-skin conditions are particularly common in the testing of ferromagnetic steel due to its high value of relative permeability.

In the thin-skin regime, the interaction of eddy currents with cracks can be described with the aid of solutions of the two-dimensional (2-D) Laplace equation in a domain which corresponds to the face of the crack. This approach overcomes the resolution problems inherent in schemes that rely on a mesh or cellular grid to represent variations in the field on the scale of a skin depth. Given that a 2-D Laplace equation is to be solved, it is necessary first to determine the boundary conditions that apply at the perimeter of the crack face, that is, at the buried edge of the crack and at the boundary where the crack intersects with the surface of the conductor, the latter being referred to as the crack mouth. These regions are shown in Fig. 1.

Manuscript received July 9, 1997; revised November 17, 1997. This work was supported by Nuclear Electric Ltd. and Magnox Electric plc, U.K.

The authors are with the Department of Physics, University of Surrey, Guildford, Surrey GU2 5XH, U.K. (e-mail: n.harfield@surrey.ac.uk).

Publisher Item Identifier S 0018-9464(98)01478-2.

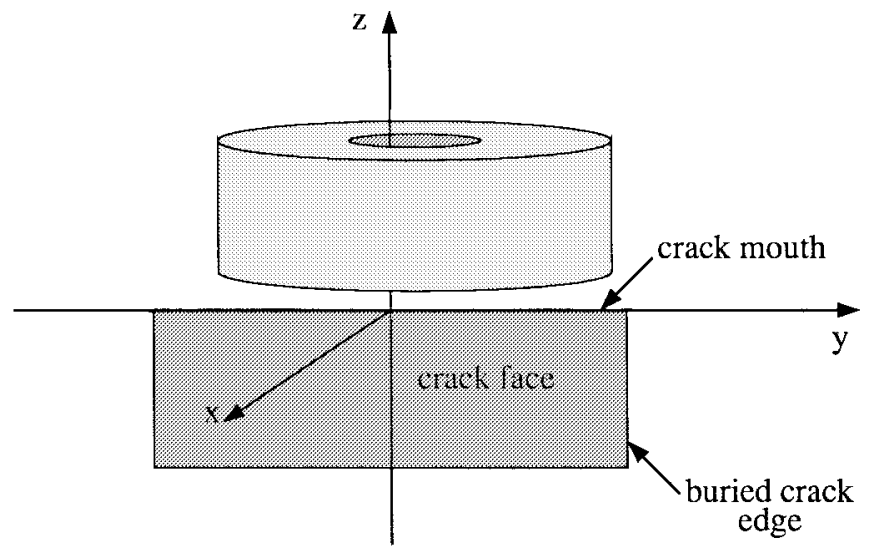

Fig. 1. A normal coil over a surface-breaking crack in a conductor. The crack is modeled as a rectangular region.

The boundary conditions on the surface Laplacian have been considered by Auld [1] for calculations of the probe impedance due to cracks in nonmagnetic materials and by Collins $e t$ al. [2] in a related problem involving cracks in ferromagnetic materials. Auld adopted an approximation which equates the total magnetic field transverse to the conductor surface at the mouth of the crack with the unperturbed field. This implies that the contribution to the tangential magnetic field at the crack mouth due to perturbations by the crack itself is negligible. Although Auld's approximation leads to satisfactory predictions, a useful advance on this theory could be made if the effect of the perturbed field is included in the mouth boundary condition. In the approach presented here, the problem is formulated without recourse to Auld's approximation.

An alternative thin-skin crack theory, intended for ferromagnetic materials, has been developed over a number of years by Collins et al. [2]. Rather than using a boundary condition at the crack mouth, a continuity condition is proposed whereby the field on the surface of the conductor is matched with that on the face of the crack. This implies that the domain of the problem does not consist of the crack face alone. Instead, the problem domain includes the half-plane representing one-half of the surface of the conductor joined to one crack face at a line of intersection known as the fold line. The crack face is unfolded into the plane of the conductor surface and a solution of the Laplace equation found in the domain of the unfolded crack face and adjoining half-plane. It is assumed that the normal current and the normal magnetic flux are continuous at the fold line, and consequently, a complex potential describing the surface field is analytically continuous at the line. The assumption that the normal flux in a thin skin 
flows continuously from the surface of the conductor to the face of the crack is acceptable provided that the permeability is high and that there is a finite gap between the crack faces. If the permeability is not sufficiently high and the gap between the crack faces is small, then there will be a tendency for the flux to flow across the crack rather than around it. Hence the conditions required for analytic continuation represent a limiting case of a more general theory.

Thin-skin eddy-current interaction with cracks in materials of arbitrary permeability were considered by Lewis et al. [3] who derived a mouth boundary condition using a conservation of flux argument and the surface impedance boundary condition. Here an approach is taken which does not make thin-skin assumptions at the outset, but establishes the required boundary condition at the crack mouth as a limiting case of a theory applicable at arbitrary frequency and skin depth. The results are comparable with, but differ in detail from, those of Lewis et al. [3].

\section{SCALAR FORMULATION}

Consider an induction coil which excites eddy currents in a half-space conductor containing a surface crack, as shown in Fig. 1. The problem is one of determining the electromagnetic field distribution and the change in the coil impedance due to the presence of this defect. An integral equation for the field at the crack is described in this section, in which the excitation frequency and hence the skin depth is arbitrary. A similar integral equation to the one used here has been discussed in earlier work, based on a magnetic vector potential representation of the field [4]. In the present study, essentially the same equation is applied in a formulation that involves a scalar decomposition of the field. Numerical solutions could be found for arbitrary frequencies, but our aim is to take the analysis further using thin-skin approximations. This is achieved by formulating the problem in terms of a potential which obeys a surface Laplace equation on the crack. The integral equation is then used to provide a thin-skin boundary condition on the solution at the crack mouth.

\section{A. Surface Potential}

In this calculation it is assumed that the material properties are linear and that the displacement current is negligible. It is also assumed that the crack has a small opening, but initially the opening will be neglected as we consider a crack defined on an open surface $S_{0}$ acting as an impenetrable barrier to the flow of current. For this idealized crack, the condition

$$
\boldsymbol{E}(\boldsymbol{r}) \cdot \hat{x}=0, \quad \boldsymbol{r} \in S_{0}
$$

applies at each and every point on the crack surface $S_{0}$.

The electromagnetic field in the conductor, varying as the real part of $\exp (-i \omega t)$, is calculated via decomposition into transverse electric (TE) and transverse magnetic (TM) components [5] and defined with respect to a preferred direction normal to the surface of the crack

$$
\begin{aligned}
& \boldsymbol{E}(\boldsymbol{r})=i \omega \mu_{0} \mu_{r}\left[\nabla \times \hat{x} \psi^{\prime}(\boldsymbol{r})-\nabla \times \nabla \times \hat{x} \psi^{\prime \prime}(\boldsymbol{r})\right] \\
& \boldsymbol{H}(\boldsymbol{r})=\nabla \times \nabla \times \hat{x} \psi^{\prime}(\boldsymbol{r})-k^{2} \nabla \times \hat{x} \psi^{\prime \prime}(\boldsymbol{r}), \quad z<0
\end{aligned}
$$

where $k^{2}=i \omega \mu_{r} \mu_{0} \sigma, \hat{x}$ is a unit vector in the preferred direction normal to the crack plane, $\psi^{\prime}$ is the TE potential, and $\psi^{\prime \prime}$ is the TM potential. As discussed in [6], the transverse electric field does not interact directly with an ideal crack which means that the scattering process can be described in terms of the transverse magnetic scalar potential alone. Applying (1) and (2) gives

$$
\nabla_{x}^{2} \psi^{\prime \prime}(\boldsymbol{r})=0, \quad \boldsymbol{r} \in S_{0}
$$

where $\nabla_{x}^{2}$ is the Laplacian operator transverse to $x$. Note that $\psi^{\prime \prime}$ satisfies the surface Laplace equation regardless of the frequency or skin depth.

The boundary condition on $\psi^{\prime \prime}$ which is to be applied at the mouth of the crack in the thin-skin limit is determined from an integral equation applicable at arbitrary frequency. The derivation of this integral equation is given in [4] in terms of a vector potential formulation, with interpretation in terms of the TM potential given in [6]. It is found that

$$
\psi^{\prime \prime}(\boldsymbol{r})=\psi^{\prime \prime(i)}(\boldsymbol{r})-\frac{1}{k^{2}} \int_{S_{c}} G\left(\boldsymbol{r}, \boldsymbol{r}^{\prime}\right) p\left(\boldsymbol{r}^{\prime}\right) d \boldsymbol{r}^{\prime}, \quad \boldsymbol{r} \in S_{0}
$$

where $p$ is an equivalent current dipole density at the crack [4], [7] and ( $i$ ) denotes the incident, or unperturbed, field. The Green's function for the TM potential in a half-space conductor is derived in [6] and is given by

$$
\begin{aligned}
G\left(\boldsymbol{r}, \boldsymbol{r}^{\prime}\right)= & \frac{e^{i k\left|\boldsymbol{r}-\boldsymbol{r}^{\prime}\right|}}{4 \pi\left|\boldsymbol{r}-\boldsymbol{r}^{\prime}\right|}+\frac{e^{i k\left|\boldsymbol{r}-\boldsymbol{r}^{\prime \prime}\right|}}{4 \pi\left|\boldsymbol{r}-\boldsymbol{r}^{\prime \prime}\right|} \\
& +\frac{1}{k^{2}} \frac{\partial^{2}}{\partial y^{2}} U\left(x-x^{\prime}, y-y^{\prime}, z+z^{\prime}\right)
\end{aligned}
$$

where $\boldsymbol{r}^{\prime}=\boldsymbol{r}^{\prime \prime}-2 \hat{z} z^{\prime}$ is the image point and

$$
\begin{aligned}
U(x, y, z)= & \frac{\mu_{r} k^{4}}{(2 \pi)^{2}} \int_{-\infty}^{\infty} \int_{-\infty}^{\infty} \frac{1}{u^{2}-k^{2}}\left(\frac{1}{\kappa}-\frac{\mu_{r}}{\gamma}\right) \\
& \cdot \frac{1}{\left[\left(\mu_{r}^{2}-1\right) \kappa^{2}+\kappa^{2}\right]} \\
& \cdot e^{-\gamma z+i u x+i v y} d u d v
\end{aligned}
$$

where $\gamma=\left(u^{2}+v^{2}-k^{2}\right)^{1 / 2}$ and $\kappa=\left(u^{2}+v^{2}\right)^{1 / 2}$.

\section{B. Magnetic Field}

In addition to the TM potential, the calculation of probe impedance change due to an open crack requires the value of the total magnetic field at the crack mouth. In particular, the component parallel to the line of the crack mouth is needed. We approximate the field in this region by the magnetic field at the mouth of a closed crack. This can be expressed as the sum of the field incident from the coil and an integral form of that scattered by the defect

$$
H_{y}(\boldsymbol{r})=H_{y}^{(i)}(\boldsymbol{r})+\int_{S_{c}} g\left(\boldsymbol{r}, \boldsymbol{r}^{\prime}\right) p\left(\boldsymbol{r}^{\prime}\right) d S^{\prime}, \quad \boldsymbol{r} \in S_{c} .
$$

This relationship is found from the corresponding integral equation for the electric field [7]. The half-space Green's 
function is given by

$$
\begin{aligned}
g\left(\boldsymbol{r}, \boldsymbol{r}^{\prime}\right)=\frac{\partial}{\partial z}\left[\frac{e^{i k\left|\boldsymbol{r}-\boldsymbol{r}^{\prime}\right|}}{4 \pi\left|\boldsymbol{r}-\boldsymbol{r}^{\prime}\right|}+\frac{e^{i k\left|\boldsymbol{r}-\boldsymbol{r}^{\prime \prime}\right|}}{4 \pi\left|\boldsymbol{r}-\boldsymbol{r}^{\prime \prime}\right|}\right. & \\
& \left.\quad-\frac{1}{k^{2}} \frac{\partial^{2}}{\partial y^{2}} V\left(x-x^{\prime}, y-y^{\prime}, z+z^{\prime}\right)\right]
\end{aligned}
$$

where $\boldsymbol{r}^{\prime \prime}=\boldsymbol{r}^{\prime}-2 \hat{z} z^{\prime}$ is the image point and

$$
\begin{aligned}
V(x, y, z)= & \frac{1}{k^{2}} \nabla_{x}^{2} U(x, y, z)=\frac{\mu_{r} k^{2}}{(2 \pi)^{2}} \\
& \cdot \int_{-\infty}^{\infty} \int_{-\infty}^{\infty}\left(\frac{1}{\kappa}-\frac{\mu_{r}}{\gamma}\right) \frac{1}{\left[\left(\mu_{r}^{2}-1\right) \kappa^{2}+k^{2}\right]} \\
& \cdot e^{-\gamma z+i u x+i v y} d u d v .
\end{aligned}
$$

\section{THIN-SKIN LIMIT}

\section{A. TM Potential}

As stated in (4), $\psi^{\prime \prime}$ obeys a 2-D Laplace equation defined on the open surface of the crack. In order to obtain a solution of this equation, boundary conditions at the crack edge and mouth are required. It is possible to proceed analytically if the frequency range is restricted so that thin-skin conditions apply. At the crack edge, the component of the magnetic field normal to the edge is zero in the thin-skin regime [8]. From this we deduce that

$$
\boldsymbol{H}_{\mathrm{TM}}\left(\boldsymbol{r}_{e}\right) \cdot \hat{n}=0
$$

where $\boldsymbol{r}_{e}$ denotes the coordinate of a point at the crack edge and $\hat{n}$ is an outward unit normal in the plane of the crack perpendicular to the edge. Equation (11) is satisfied by the TM component of the magnetic field [see (3)] by putting $\psi^{\prime \prime}$ equal to a constant at the edge. Hence, setting the arbitrary constant to zero, the edge boundary condition is written

$$
\psi\left(\boldsymbol{r}_{e}\right)=0
$$

where, for notational convenience, $\psi=-k^{2} \psi^{\prime \prime}$.

We will now use (5) to obtain a thin-skin boundary condition applicable at the crack mouth. The $y$-component of the transverse magnetic field at the crack mouth is expressed as

$$
H_{y \mathrm{TM}}\left(\boldsymbol{r}_{m}\right)=H_{y \mathrm{TM}}^{(i)}\left(\boldsymbol{r}_{m}\right)+H_{y \mathrm{TM}}^{(s)}\left(\boldsymbol{r}_{m}\right)
$$

where $\boldsymbol{r}_{m}$ denotes the coordinate of a point at the crack mouth. The superscripts $(i)$ and $(s)$ denote the incident field due to an eddy-current probe and the field scattered by the defect, respectively. In Auld's theory for nonmagnetic conductors [1], $H_{y \mathrm{TM}}^{(s)}\left(\boldsymbol{r}_{m}\right)$ is neglected in establishing an approximate mouth boundary condition. Here, in contrast, the scattered field at the mouth and its dependence on $\mu_{r}$ are considered.

The required boundary condition is derived from (5) by first eliminating the dipole density $p$ from the equation. In order to approximate $p$ in the thin-skin limit, note that the effective linear range of the integral operator in (5) is no more than a few skin depths. In the thin-skin regime, the effect of the integral operator becomes highly localized such that, at points away from the crack boundary, the kernel can be approximated by a delta function [4]. Hence, using (5), it can be shown that

$$
p(\boldsymbol{r}) \approx-2 i k \psi(\boldsymbol{r}) .
$$

Numerical tests have demonstrated that this relationship is a reasonable approximation at the crack mouth [4]. In the thinskin limit it is exact at points sufficiently far from the crack perimeter.

Differentiating (5) with respect to $z$, using (14) and restricting $\boldsymbol{r}$ to $\boldsymbol{r}_{m}$ gives

$$
\begin{aligned}
\left.\frac{\partial \psi(\boldsymbol{r})}{\partial z}\right|_{\boldsymbol{r}=\boldsymbol{r}_{m}}= & \left.\frac{\partial \psi^{(i)}(\boldsymbol{r})}{\partial z}\right|_{\boldsymbol{r}=\boldsymbol{r}_{m}}+\frac{2}{i k} \\
& \cdot \int_{-c / 2}^{c / 2} \frac{\partial^{2}}{\partial y^{2}} U\left(0, y-y^{\prime}, 0\right) \psi\left(0, y^{\prime}, 0\right) d y^{\prime}
\end{aligned}
$$

where $c$ is the crack length. The integration with respect to $z^{\prime}$ has been carried out assuming that $\psi\left(0, y^{\prime}, z^{\prime}\right)$ varies slowly with $z^{\prime}$ and is therefore roughly constant over the effective range of the kernel in the $z$-direction. Equations (15) and (13) are equivalent. It is now possible to solve the surface Laplace equation for $\psi$ by applying (12) and (15) at the crack edge and mouth, respectively.

The function $U(0, y, 0)$ can be represented algebraically in terms of its Fourier transform in the $y$-direction. Using the definition

$$
\tilde{U}(x, v, z)=\int_{-\infty}^{\infty} U(x, y, x) e^{-i v y} d y
$$

it can be seen from (17) that

$$
\begin{aligned}
\tilde{U}(v)= & \frac{\mu_{r} k^{4}}{2 \pi} \int_{-\infty}^{\infty} \frac{1}{u^{2}-k^{2}} \\
& \cdot\left(\frac{1}{\kappa}-\frac{\mu_{r}}{\gamma}\right) \frac{1}{\left[\left(\mu_{r}^{2}-1\right) \kappa^{2}+k^{2}\right]} d u
\end{aligned}
$$

where $\tilde{U}(v)=\tilde{U}(0, v, 0)$. The integral in (17) can be evaluated by splitting the integrand using partial fractions and using the standard form

$$
\begin{aligned}
F(\alpha, \beta) & =\int_{-\infty}^{\infty} \frac{d u}{\left(u^{2}-\alpha^{2}\right) \sqrt{u^{2}+\left(\beta^{2}-\alpha^{2}\right)}} \\
& =\frac{1}{\alpha \beta} \ln \left(\frac{\alpha-\beta}{\alpha+\beta}\right) .
\end{aligned}
$$

This gives

$$
\begin{aligned}
\tilde{U}(v)= & \frac{\mu_{r}}{2 \pi\left[\left(\mu_{r}^{2}-1\right) w^{2}+\mu_{r}^{2}\right]} \\
& \cdot\left\{\frac{\mu_{r}}{w} \ln \left(\frac{1+w}{1-w}\right)-\frac{1}{\sqrt{1+w^{2}}} \ln \left(\frac{1+\sqrt{1+w^{2}}}{1-\sqrt{1+w^{2}}}\right)\right. \\
& +\frac{\mu_{r}^{2}-1}{\sqrt{\left(\mu_{r}^{2}-1\right) w^{2}+1}} \\
& \cdot\left[\ln \left(\frac{\sqrt{\left(\mu_{r}^{2}-1\right) w^{2}+1}+\mu_{r}}{\sqrt{\left(\mu_{r}^{2}-1\right) w^{2}+1}-\mu_{r}}\right)\right. \\
& \left.\left.\quad-\ln \left(\frac{\sqrt{\left(\mu_{r}^{2}-1\right) w^{2}+1}+1}{\sqrt{\left(\mu_{r}^{2}-1\right) w^{2}+1}-1}\right)\right]\right\}
\end{aligned}
$$

where $w=v / k$. 


\section{B. Magnetic Field}

The thin-skin relation between $p$ and $\psi$ given in (14) can also be used with (8) to give $H_{y}$ at the crack mouth, which is required in the calculation of probe impedance change due to open cracks

$$
\begin{aligned}
H_{y}\left(\boldsymbol{r}_{m}\right)= & H_{y}^{(i)}\left(\boldsymbol{r}_{m}\right)-\frac{2}{i k} \int_{-c / 2}^{c / 2} \frac{\partial^{2}}{\partial y^{2}} \\
& \cdot V\left(0, y-y^{\prime}, 0\right) \psi\left(0, y^{\prime}, 0\right) d y^{\prime} .
\end{aligned}
$$

Again, the integration with respect to $z^{\prime}$ has been carried out by assuming that $\psi^{\prime \prime}\left(0, y^{\prime}, z^{\prime}\right)$ varies slowly with $z^{\prime}$ and is therefore roughly constant over the effective range of the kernel in the $z$-direction.

The kernel $V$ in the integral of (20) can be treated similarly to $U$ in (15), to obtain an algebraic representation in terms of its Fourier transform in $y$

$$
\tilde{V}(x, v, z)=\int_{-\infty}^{\infty} V(x, y, z) e^{-i v y} d y
$$

From (10) we have, putting $\tilde{V}(v)=\tilde{V}(0, v, 0)$

$$
\tilde{V}(v)=\frac{\mu_{r} k^{2}}{2 \pi} \int_{-\infty}^{\infty}\left(\frac{1}{\kappa}-\frac{\mu_{r}}{\gamma}\right) \frac{1}{\left[\left(\mu_{r}^{2}-1\right) \kappa^{2}+k^{2}\right]} d u
$$

which can be integrated to give

$$
\begin{aligned}
\tilde{V}(v)= & -\frac{\mu_{r}}{2 \pi} \\
& {\left[\ln \left(\frac{\sqrt{\left(\mu_{r}^{2}-1\right) w^{2}+1}+\mu_{r}}{\sqrt{\left(\mu_{r}^{2}-1\right) w^{2}+1}-\mu_{r}}\right)-\frac{1}{\sqrt{\left(\mu_{r}^{2}-1\right) w^{2}+1}}\right.} \\
& \left.\cdot \ln \left(\frac{\sqrt{\left(\mu_{r}^{2}-1\right) w^{2}+1}+1}{\sqrt{\left(\mu_{r}^{2}-1\right) w^{2}+1}-1}\right)\right] .
\end{aligned}
$$

Simplified limiting forms of $\tilde{U}$ and $\tilde{V}$ have been discussed elsewhere [6].

\section{Probe Impedance}

An eddy-current coil placed near the surface of a conductor has a certain value of impedance. If a crack appears in the conductor, then the value of the coil impedance changes and this change, denoted $\Delta Z$, is the impedance change in the probe due to the flaw. As shown in [6], the impedance change in the thin-skin regime can be expressed in terms of one-dimensional (1-D) integrals along the line of the crack mouth by using the reciprocity theorem and the surface impedance boundary condition. The impedance change is expressed as

$$
\Delta Z=Z_{F}+Z_{S}
$$

where

$$
\begin{aligned}
Z_{F}= & -\frac{1}{\sigma}\left(k^{2} a \frac{\mu_{c}}{\mu_{r}}+2 i k\right) \int_{-c / 2}^{c / 2} H_{y}^{(i)}(0, y, 0) \psi(0, y, 0) d y \\
& -\frac{i k a}{\sigma} \int_{-c / 2}^{c / 2} H_{y}^{(i)}(0, y, 0) H_{t \mathrm{TM}}\left(0, y, z_{e}\right) d y
\end{aligned}
$$

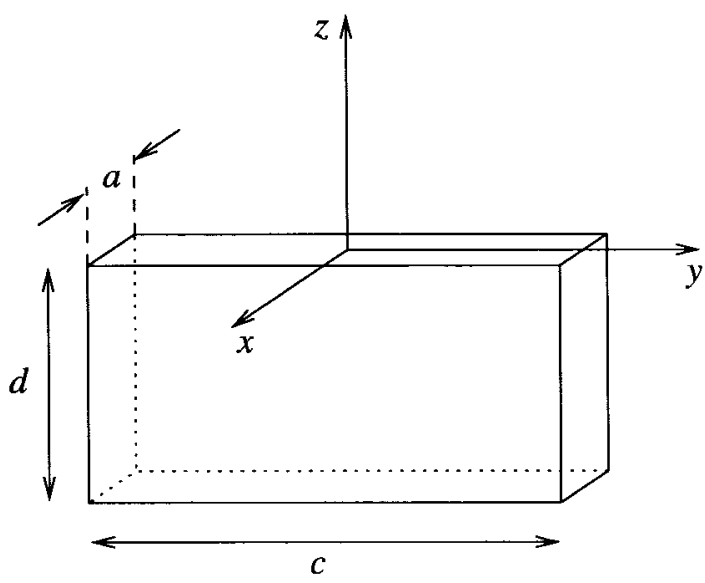

Fig. 2. Dimensions of a rectangular surface slot.

and

$$
Z_{S}=\frac{i k a}{\sigma} \int_{-c / 2}^{c / 2} H_{y}^{(i)}(0, y, 0) H_{y}(0, y, 0) d y .
$$

In (25) and (26), $a$ is the distance between the crack faces (the gape), $c$ is the crack length, and $\mu_{c}$ is the relative permeability of material filling the crack. The dimensions of a rectangular slot are shown in Fig. 2. The subscript $t$ in $H_{t \mathrm{TM}}$ indicates that the component of $H_{\mathrm{TM}}$ tangential to the line of the crack edge is required. $Z_{F}$ represents the impedance change due to the presence of the crack volume, faces, and base, and $Z_{S}$ represents the impedance change due to the loss of conductor surface on introduction of the crack. It is assumed that the gape of the defect is uniform over its entire depth, and it can be clearly seen that the loss of surface term $Z_{S}$ is balanced by the second term in $Z_{F}$ which represents the effect of the defect base. These contributions are of opposite sign since one represents loss of conductor surface and the other represents introduction of surface. The first term in $Z_{F}$ consists of a contribution of order $k^{2}$ and is related to the crack volume. The contribution of order $i k$ represents the impedance change due to the presence of the crack faces. As described in [6], it is possible to calculate, approximately, contributions to $\Delta Z$ of order $k^{0}$. These will not be considered here, however, since in practice the contribution to $|\Delta Z|$ from terms of this order is typically only about $5 \%$ even for defects only $3 \delta$ deep.

For a coil whose axis is normal to the conductor surface, the incident magnetic field required in the integrands of (25) and (26) can be calculated using closed-form expressions [9]. $H_{y}$ and $\psi$ are calculated as described in the previous section and $H_{t \mathrm{TM}}$ is determined from $\psi$.

\section{EXAMPLE SOLUTION}

Consider a rectangular crack of length $c$ and depth $d$ whose normal is in the positive $x$ direction. The TM potential required in the evaluation of (25) is written as the following solution of the 2-D Laplace equation:

$$
\psi(y, z)=\sum_{n} C_{n} \frac{c \sin [(n \pi / 2)(2 y / c+1)] \sinh [n \pi(d+z) / c]}{n \pi \cosh (n \pi d / c)} .
$$


TABLE I

CoIl PARAmeters

\begin{tabular}{lcc}
\hline \hline coil & C7 & C9 \\
\hline inner radius $/ \mathrm{mm}$ & 6.95 & 3.015 \\
outer radius $/ \mathrm{mm}$ & 9.35 & 5.46 \\
lift-off/mm & 2.07 & 1.29 \\
length $/ \mathrm{mm}$ & 6.70 & 2.94 \\
no. of turns & 335 & 900 \\
free-space inductance/mH & 1.752 & 6.027 \\
\hline \hline
\end{tabular}

Equation (27) vanishes for $y= \pm c / 2$ with $0 \geq z \geq-d$ and for $z=-d$ with $|y| \leq c / 2$ as required. Note that

$$
\begin{gathered}
\left.\frac{\partial \psi(y, z)}{\partial z}\right|_{z=0}=\sum C_{n} \sin [(n \pi / 2)(2 y / c+1)] \\
|y| \leq c / 2 .
\end{gathered}
$$

The expansion coefficients $C_{n}$ are determined by substituting (28) into (15), multiplying by $\sin [(m \pi / 2)(2 y / c+1)]$, and integrating from $-c / 2$ to $c / 2$. This gives

$$
C_{m}^{(i)}=\sum_{n}\left[\delta_{m n}+\frac{2}{i k n \pi} U_{m n} \tanh (n \pi \beta)\right] C_{n}
$$

where $\beta=d / c$, the expansion coefficients $C_{m}^{(i)}$ are defined such that

$$
H_{y \mathrm{TM}}^{(i)}=\left.\frac{\partial \psi^{(i)}(y, z)}{\partial z}\right|_{z=0}=\sum_{|y| \leq c / 2} C_{n}^{(i)} \sin [(n \pi / 2)(2 y / c+1)]
$$

and matrix elements $U_{m n}$ have been introduced defined by

$$
\begin{aligned}
U_{m n}= & -2 \int_{-c / 2}^{c / 2} \sin [(m \pi / 2)(2 y / c+1)] \int_{-c / 2}^{c / 2} \frac{\partial^{2}}{\partial y^{2}} \\
& \cdot U\left(0, y-y^{\prime}, 0\right) \sin \left[(n \pi / 2)\left(2 y^{\prime} / c+1\right)\right] d y d y^{\prime} .
\end{aligned}
$$

The evaluation of the matrix elements is carried out using the Fourier representation

$$
U(0, y, 0)=\frac{1}{2 \pi} \int_{-\infty}^{\infty} \tilde{U}(v) e^{i v y} d v
$$

where $\tilde{U}(v)$ is given by (19). Substituting (32) into (31) gives

$$
\begin{aligned}
U_{m n}= & \frac{c^{2}}{\pi} \int_{-\infty}^{\infty} v^{2} \tilde{U}(v) F_{m}(c v / 4) F_{n}(-c v / 4) d v \\
= & \frac{c^{2}}{\pi} \int_{0}^{\infty} v^{2} \tilde{U}(v) \\
& \cdot\left[F_{m}(c v / 4) F_{n}(-c v / 4)+F_{m}(-c v / 4) F_{n}(c v / 4)\right] d v
\end{aligned}
$$

where $F_{m}(s)$ is given by

$$
\begin{aligned}
F_{m}(s) & =\int_{0}^{1} \sin (m \pi w) e^{i s w} d w \\
& =\frac{1-e^{i(s+m \pi)}}{2(s+m \pi)}-\frac{1-e^{i(s-m \pi)}}{2(s-m \pi)} .
\end{aligned}
$$

It is now a straightforward matter to calculate the value of $\psi(y, 0)$ from (27) by solving the matrix (29) for the coefficients $C_{n}$.
TABLE II

Material and Defect Parameters for Rectangular Slots in Aluminum and Mild Steel

\begin{tabular}{lccc}
\hline \hline defect & 1 & 2 & 3 \\
\hline depth $/ \mathrm{mm}$ & 5.0 & 2.9 & 1.89 \\
length $/ \mathrm{mm}$ & 12.6 & 47.1 & 20.1 \\
gape $/ \mathrm{mm}$ & 0.28 & 0.64 & 0.62 \\
\hline \hline material & \multicolumn{2}{c}{ aluminium } & mild steel \\
\hline conductivity $/ \mathrm{Sm}^{-1}$ & $3.0581 \times 10^{7}$ & $1.6667 \times 10^{7}$ & $5.587 \times 10^{6}$ \\
relative permeability & 1 & 1 & $85 \pm 5$ \\
\hline \hline
\end{tabular}

According to (26), the $y$-component of the magnetic field at the crack mouth is also required in the calculation of probe impedance change due to an open crack. Expressing the surface field as the following expansion:

$$
H_{y}(y, 0)=\sum_{n} D_{n} \sin [(n \pi / 2)(2 y / c+1)]
$$

the coefficients $D_{n}$ can be found using (20). Making the thin-skin approximation ${ }^{1}$

$$
H_{y \mathrm{TM}}^{(i)} \approx H_{y}^{(i)}
$$

(30) can be substituted into (20) for $H_{y}^{(i)}$. Also substituting (35) and (27) into (20), multiplying by $\sin [(m \pi / 2)(2 y / c+1)]$ and integrating from $-c / 2$ to $c / 2$ gives

$$
D_{m}=C_{m}^{(i)}-\frac{2}{i k \pi} \sum_{n} \frac{V_{m n}}{n} \tanh (n \pi \beta) C_{n}
$$

where, in parallel with (31)

$$
\begin{aligned}
V_{m n}= & -2 \int_{-c / 2}^{c / 2} \sin [(m \pi / 2)(2 y / c+1)] \\
& \cdot \int_{-c / 2}^{c / 2} \frac{\partial^{2}}{\partial y^{2}} V\left(0, y-y^{\prime}, 0\right) \\
& \cdot \sin \left[(n \pi / 2)\left(2 y^{\prime} / c+1\right)\right] d y d y^{\prime} .
\end{aligned}
$$

Finally, as in the above analysis for $U_{m n}$, we can write

$$
\begin{aligned}
V_{m n}= & \frac{c^{2}}{\pi} \int_{0}^{\infty} v^{2} \tilde{V}(v) \\
& \cdot\left[F_{m}(c v / 4) F_{n}(-c v / 4)+F_{m}(-c v / 4) F_{n}(c v / 4)\right] d v
\end{aligned}
$$

where $F_{m}(s)$ is given by (34) and $\tilde{V}(v)$ is given in (23). The value of $H_{y}(y, 0)$ required for evaluation of (26) is now given by (35) with (37) and (39).

The probe impedance change can now be evaluated by approximating $H_{y}^{(i)}$ in (25) and (26) by $H_{y \mathrm{TM}}^{(i)}$ [see relation (36)] and using the series expansion given in (30). Since $\psi(0, y)$ is also represented as a series expansion in (27), orthogonality then reduces the integrals of (25) and (26) to sums of products of the coefficients occurring in the series.

$$
\begin{aligned}
& { }^{1} \text { It can be shown that } \\
& \qquad \tilde{\tilde{H}}_{y \mathrm{TM}}^{(i)}(u, v, z)=\frac{k^{2}}{k^{2}-u^{2}} \tilde{\tilde{H}}_{y}^{(i)}(u, v, z)
\end{aligned}
$$

where $\tilde{\tilde{H}}_{y \mathrm{TM}}^{(i)}(u, v, z)$ is the 2-D Fourier transform of $H_{y \mathrm{TM}}^{(i)}(x, y, z)$. In the thin-skin limit, the spatial frequency $u$ is typically much smaller than $k$. Putting $u^{2} \ll k^{2}$ in the above gives (36). 


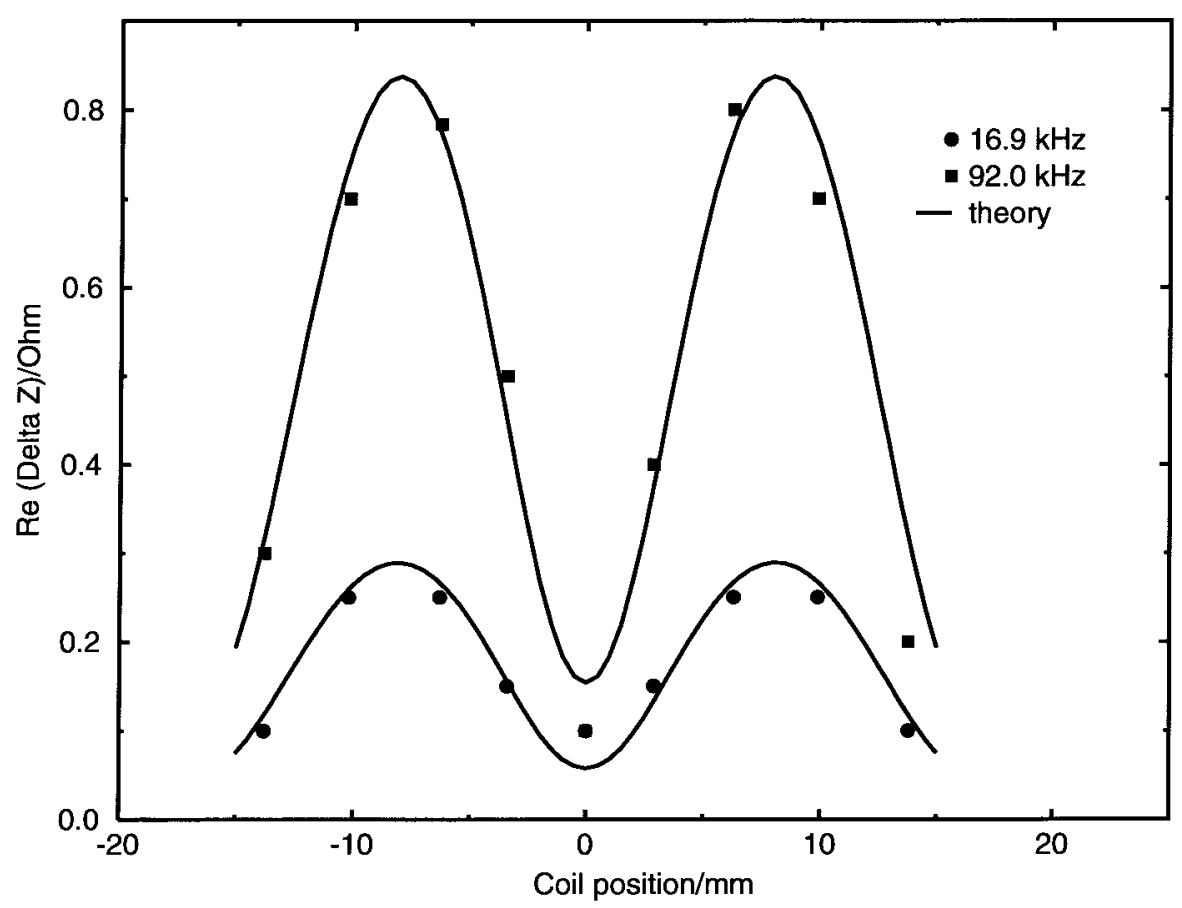

Fig. 3. The real part of the impedance change in coil C7 as it is scanned along the mouth of rectangular slot 1 (aluminum).

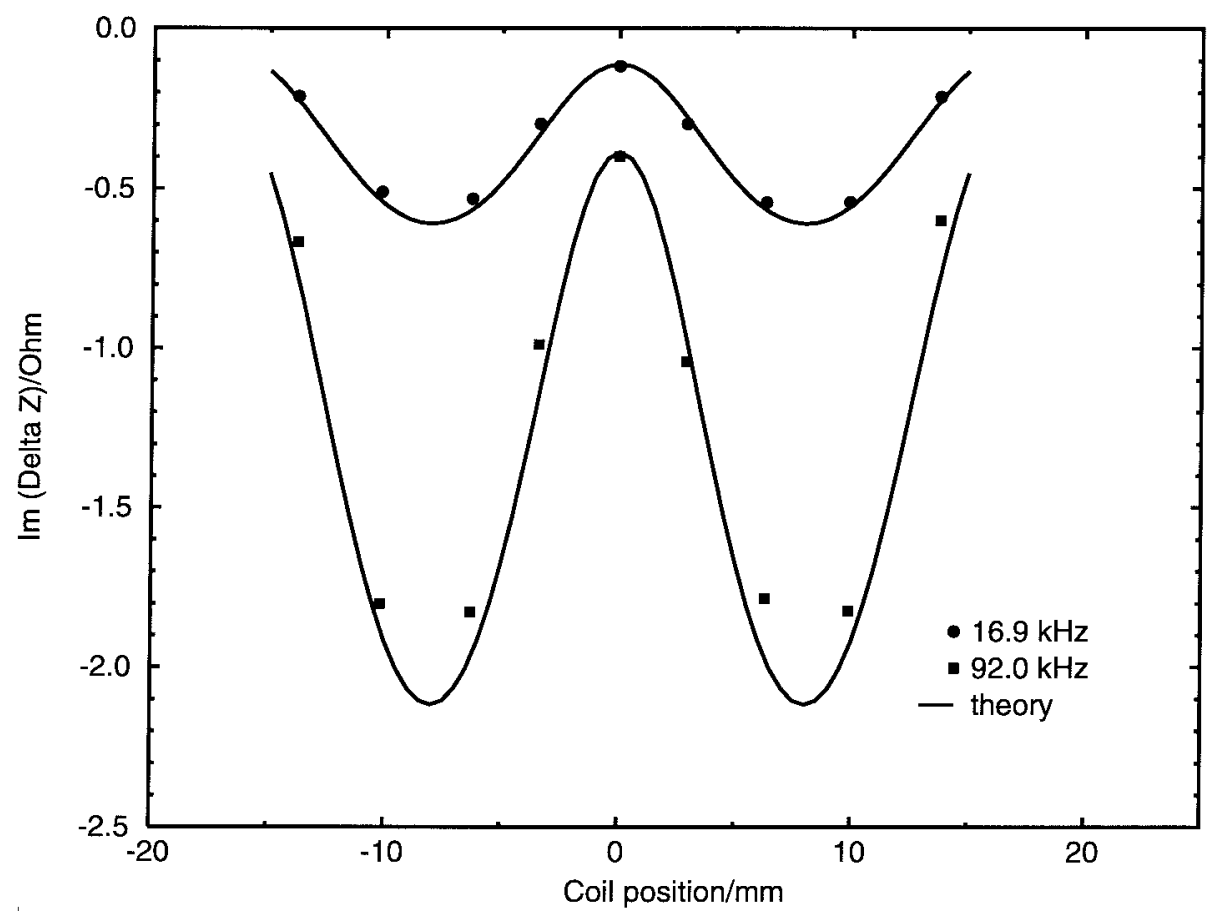

Fig. 4. The imaginary part of the impedance change in coil C7 as it is scanned along the mouth of rectangular slot 1 (aluminum).

\section{Predictions and Experimental Data}

In Figs. 3-8, predictions of the change in coil impedance are compared with experimental data for coils scanned along the mouths of rectangular slots in aluminum and mild steel. While practical eddy-current inspections search for fatigue cracks, it is valuable to perform controlled experiments using wellcharacterized slots, even though fatigue cracks are typically subject to additional mechanical stresses. For each slot, the impedance change is shown at two frequencies. The coil parameters are given in Table I and the material and defect parameters are given in Table II.

Two sets of comparisons between theory and experiment are presented for slots in aluminum in order to test the theory for different ratios of coil diameter to slot length. In Figs. 3 and 4 , the coil diameter is greater than the slot length, whereas in Figs. 5 and 6, the slot length is about twice the diameter of the coil. The predictions well reflect the shapes described by the data points in both of these cases. The magnitude 


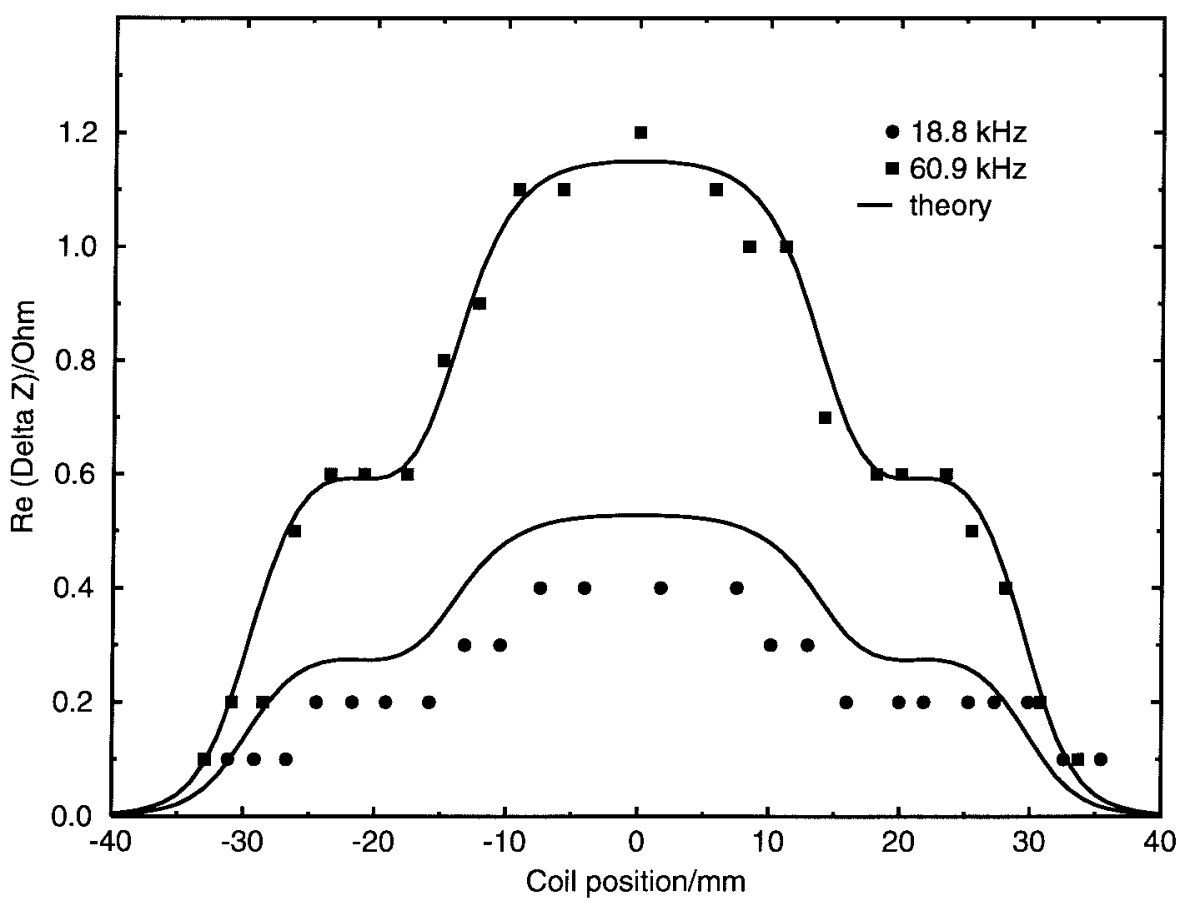

Fig. 5. The real part of the impedance change in coil C7 as it is scanned along the mouth of rectangular slot 2 (aluminum).

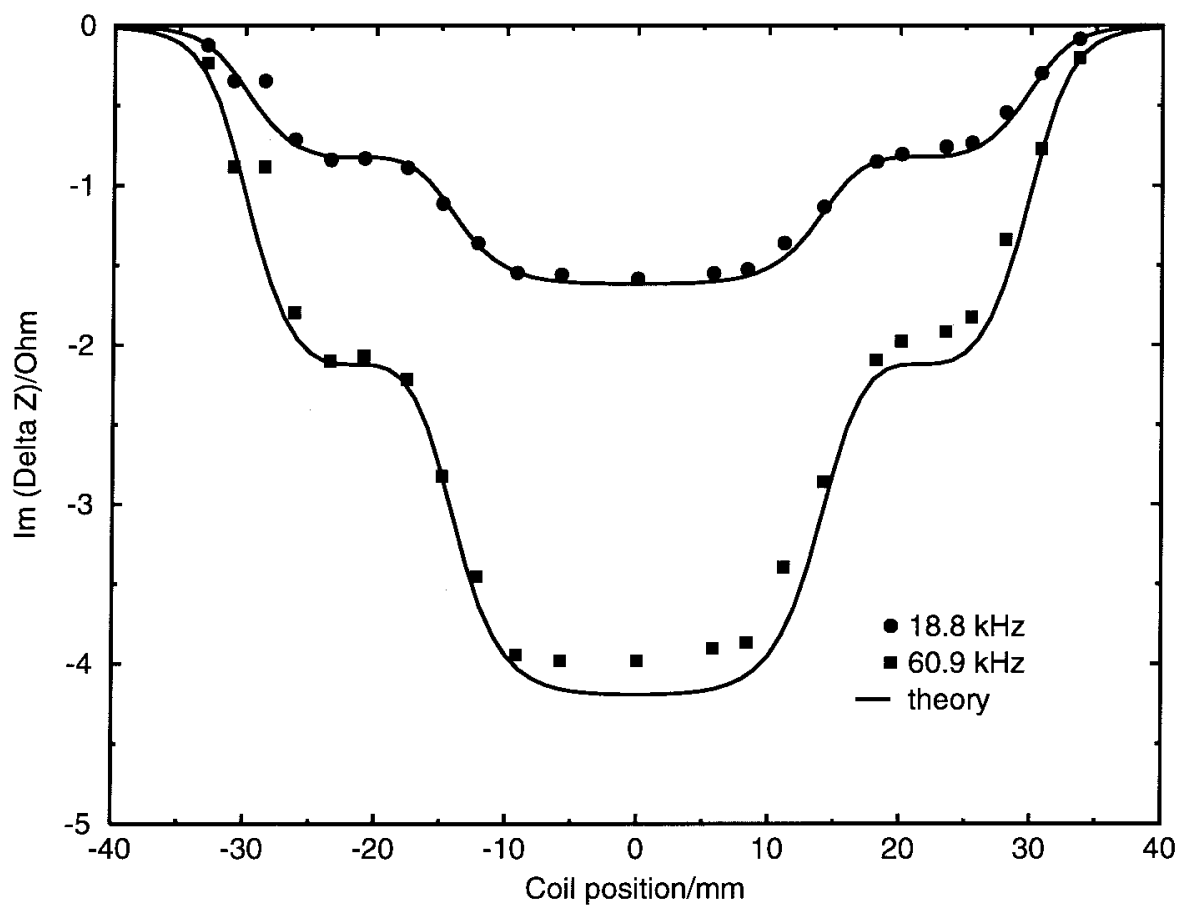

Fig. 6. The imaginary part of the impedance change in coil C7 as it is scanned along the mouth of rectangular slot 2 (aluminum).

of the imaginary part of the impedance predictions matches extremely well with the experimental data for both frequencies shown here (and down to frequencies where the defect is only about three skin depths deep). The real part matches well for the higher frequency shown, but not so well at the lower frequency where the thin-skin theory is starting to break down. However, the error in $|\Delta Z|$ is only about $5 \%$ even for slots three skin-depths deep.

In Figs. 7 and 8, theoretical predictions of coil impedance change are compared with experimental data for a slot in mild steel. Again, the predictions well reflect the shape described by the data points. Predictions of $|\Delta Z|$ and experimental data agree to within $15 \%$ for both frequencies shown. The larger error observed between predictions and experiment in the case of mild steel is likely to be a consequence of the magnetic nature of the steel. First, the relative permeability is measured in a region of the plate, well away from the defect, whereas the eddy currents at the crack faces flow in a region in which $\mu_{r}$ is likely to differ from the bulk value as a result of surface changes brought about in the manufacture of the slot. Second, 


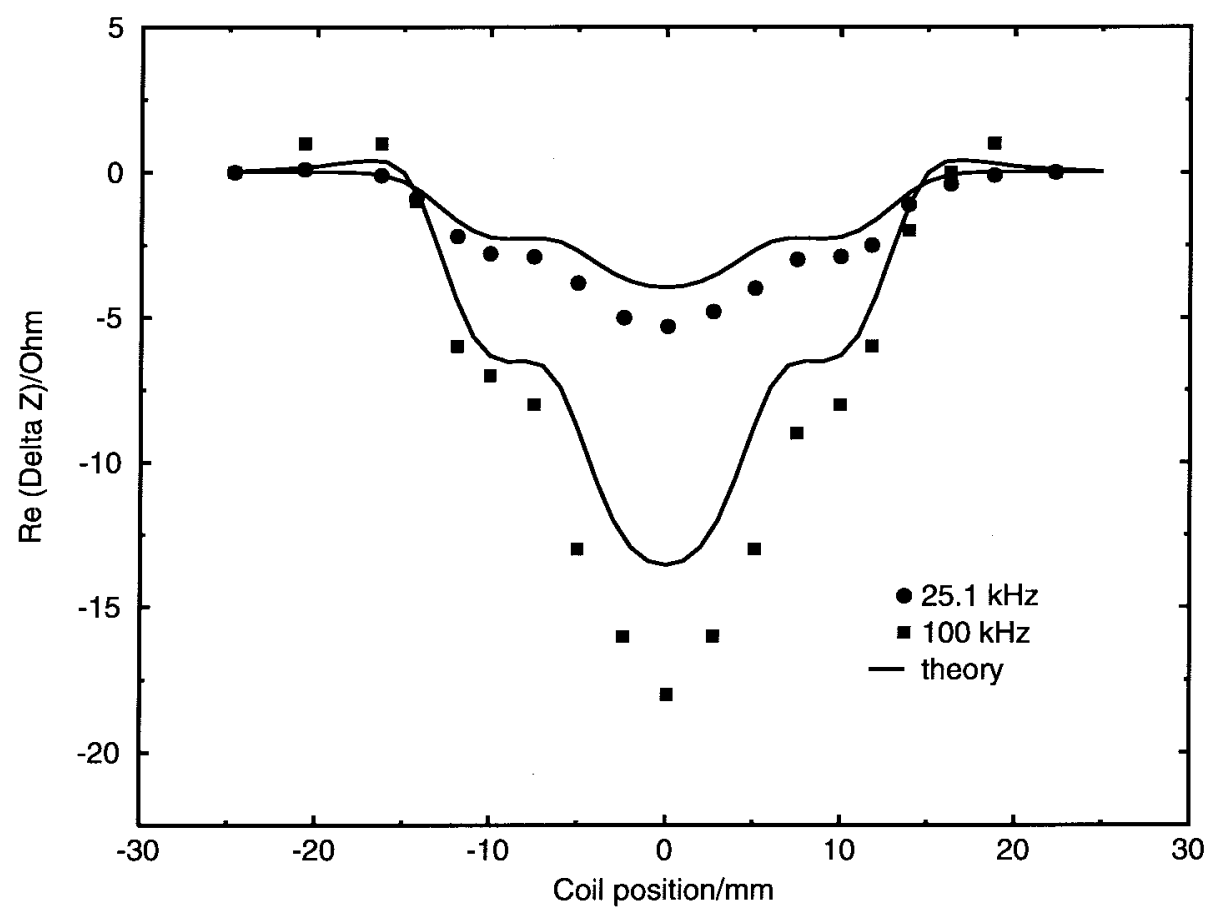

Fig. 7. The real part of the impedance change in coil C9 as it is scanned along the mouth of rectangular slot 3 (mild steel).

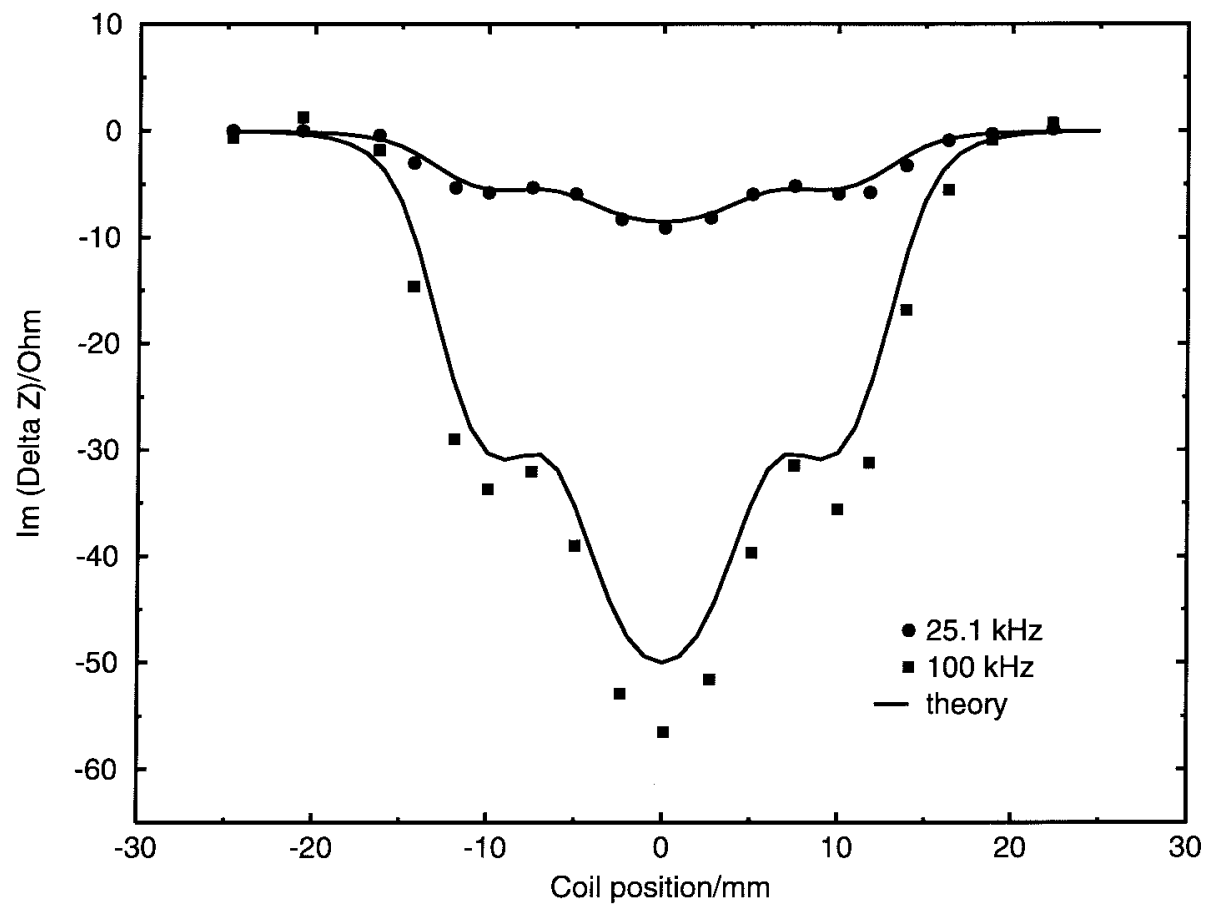

Fig. 8. The imaginary part of the impedance change in coil C9 as it is scanned along the mouth of rectangular slot 3 (mild steel).

it is possible that such surface changes give rise to a larger "effective" defect volume, which leads to impedance changes larger than those predicted.

\section{CONCLUSION}

A number of general-purpose numerical methods are available for predicting the probe signals encountered in eddy-current nondestructive evaluation. These include finiteelement, boundary-element, finite-difference, and volumeelement schemes. All of these approaches can be particularly time consuming if the skin depth of the induced current is small compared with the crack size. The reason for this is simply that general numerical schemes typically rely on a grid of cells or a nodal mesh with a density that can be adjusted to suit the length scale of the characteristic field variations. In a high-frequency eddy-current problem, where the skin depth is small compared with the defect size, the number of cells or nodes may have to be extremely large in order to represent the field properly. As a result, computation times can become very large and results may be unreliable. In this 
work, the thin-skin electromagnetic fields at the crack are described in terms of solutions of the 2-D Laplace equation. This approach overcomes the resolution difficulties inherent in the schemes mentioned above and is computationally efficient, taking only a few seconds on a personal computer for a typical calculation. The theory is valid for both magnetic and nonmagnetic materials and very good agreement between predictions and experimental data is shown for rectangular slots in aluminum and steel.

\section{ACKNOWLEDGMENT}

The authors would like to thank Dr. S. K. Burke of the DSTO Aeronautical and Maritime Research Laboratory, Melbourne, Australia, who supplied the experimental data.

\section{REFERENCES}

[1] B. A. Auld, F. G. Muennemann, and M. Riaziat, "Quantitative modeling of flaw responses in eddy current testing," in Research Techniques in Nondestructive Testing, vol. 7, R. S. Sharpe, Ed. London: Academic, 1984, pp. 37-76.
[2] R. Collins, W. D. Dover, and D. H. Michael, "The use of AC field measurements for nondestructive testing," in Research Techniques in Nondestructive Testing, vol. 8, R. S. Sharpe, Ed. London: Academic, 1985, pp. 211-267.

[3] A. M. Lewis, D. H. Michael, M. C. Lugg, and R. Collins, "Thinskin electromagnetic fields around surface-breaking cracks in metals," J. Appl. Phys., vol. 64, pp. 3777-3784, Oct. 1988.

[4] J. R. Bowler, Y. Yoshida, and N. Harfield, "Vector potential boundary integral formulation for the evaluation of eddy current interaction with a crack," IEEE Trans. Magn., vol. 33, pp. 4287-4294, Sept. 1997.

[5] L. B. Felsen and N. Marcuvitz, Radiation and scattering of waves. Englewood Cliffs, NJ: Prentice-Hall, 1973.

[6] N. Harfield and J. R. Bowler, "Theory of thin-skin eddy-current interaction with surface cracks," J. Appl. Phys., vol. 82, pp. 4590-4603, Nov. 1997.

[7] J. R. Bowler, "Eddy-current interaction with an ideal crack. I. The forward problem," J. Appl. Phys., vol. 75, pp. 8128-8137, June 1994.

[8] A. M. Lewis, "A theoretical model of the response of an eddy-current probe to a surface-breaking metal fatigue crack in a flat test-piece," $J$. Phys. D, Appl. Phys., vol. 25, pp. 319-326, 1992.

[9] C. V. Dodd and W. E. Deeds, "Analytical solutions to eddy-current probe-coil problems," J. Appl. Phys., vol. 39, pp. 2829-2838, May 1968. 